\title{
A comparison between Hydrochloric acid and Trifluoroacetic acid in hydrolysis method of exopolysaccharide from Ophiocordyceps sinensis in Monosaccharide composition analysis by GC-FID
}

\author{
Le Thi Thuy Hang ${ }^{1,2,3^{*}}$, Ha Bao Chau ${ }^{4}$, Tran Van Hai Nam ${ }^{4}$, Pham Minh Thong ${ }^{4}$, Dang \\ Hoang $\mathrm{Phu}^{5}$, Dinh Minh Hiep ${ }^{6}$, Nguyen Tien Thang ${ }^{1,2}$ \\ ${ }^{1}$ Institute of Tropical Biology, Vietnam Academy of Science and Technology, Vietnam \\ ${ }^{2}$ Graduate University of Science and Technology, Vietnam Academy of Science and \\ Technology, Vietnam \\ ${ }^{3}$ Faculty of Food Technology, Ho Chi Minh City University of Food Industry, Vietnam \\ ${ }^{4}$ Faculty of Biology \& Biotechnology, VNUHCM-University of Science, Vietnam \\ ${ }^{5}$ Department of Organic Chemistry, Faculty of Chemistry, VNUHCM-University of Science, \\ Ho Chi Minh, Vietnam \\ ${ }^{6}$ Ho Chi Minh City Agricultural Hi-Tech Park, Ho Chi Minh City, Vietnam \\ *Corresponding author: hangltt@cntp.edu.vn
}

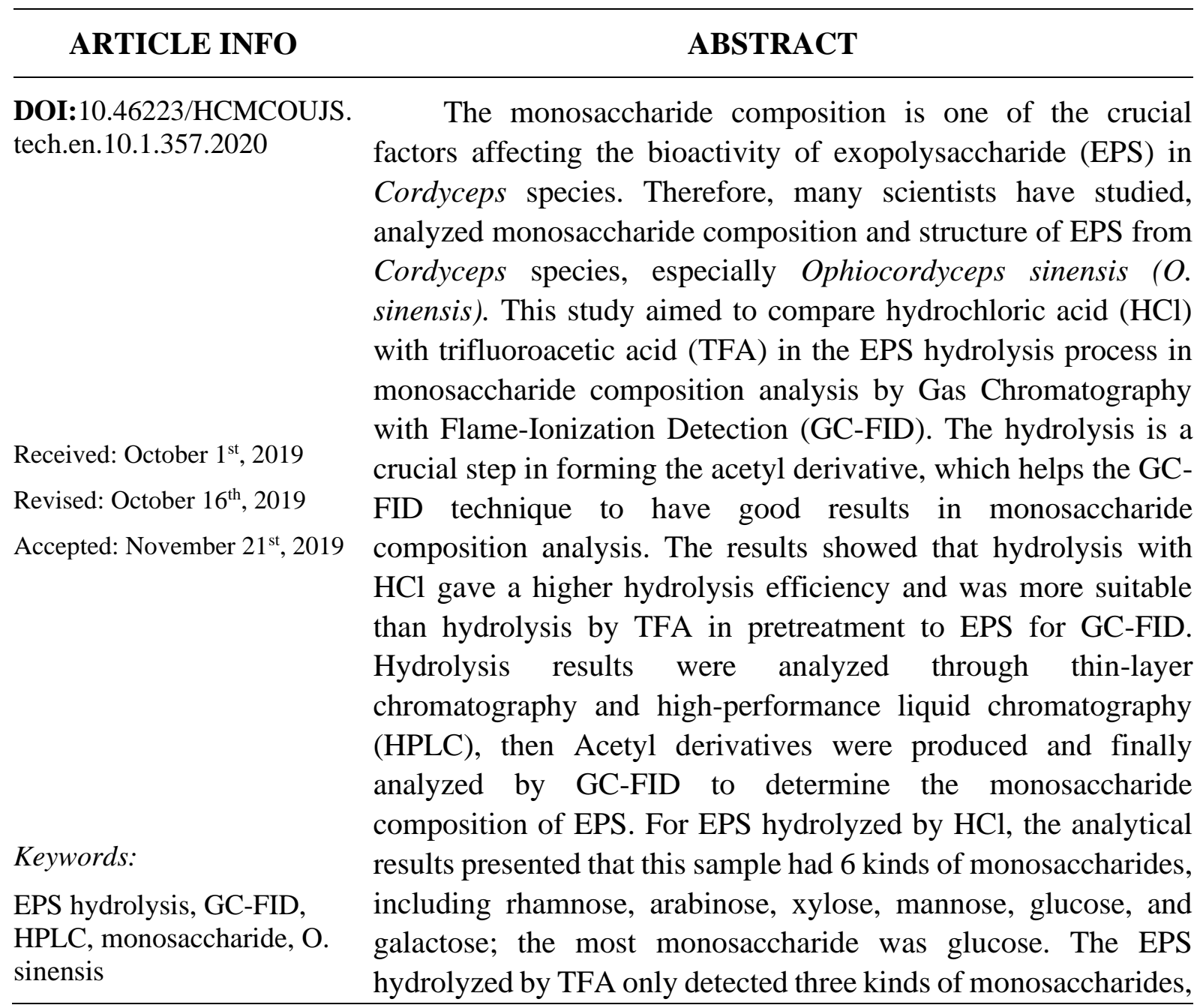


including mannose, arabinose, and galactose, mainly mannose. The study has set a foundation for further analysis of monosaccharide composition and structure of EPS from $O$. sinensis.

\section{Introduction}

Ophiocordyceps sinensis (O. sinensis), or cordyceps mushroom, is an insect parasitic fungus (Lo, Hsieh, Lin, \& Hsu, 2013). It has been found in abundance on the Qinghai - Tibet Plateau, used to enhance health and supported the treatment of many conditions and diseases (Nie, Cui, \& Xie, 2018). Exopolysaccharide (EPS), a natural source of pharmaceutical substances, has possessed promising activities such as immunomodulation, inhibition of tumor formation, and metastases that help support cancer treatment. These biological activities have been studied and applied widely in fields such as pharmaceuticals, nutraceuticals, functional foods, and cosmeceuticals (Yan, Wang, \& Wu, 2014). Antioxidants of EPS can support the prevention of related diseases such as diabetes, atherosclerosis, nephritis, Alzheimer's disease. The precious bioactivities of EPS have been proven to relate to the ratio of monosaccharides (Soltani, Kamyab, \& El-Enshasy, 2013). Therefore, determining the composition of monosaccharides is a necessary activity to predict and improve the efficiency of EPS from $O$. sinensis.

To determine the composition of monosaccharides, EPS is usually hydrolyzed to simple sugar molecules by acid (Selvendran, March, \& Ring, 1979). Monosaccharide molecules will continue to be modified to suit analytical techniques such as gas-liquid chromatography or Gas chromatograph-mass spectrometry (Merkle \& Poppe, 1994). The effect of each acid on the same EPS sample is complex so that the analysis results are different in quality (Hon, 2001; Merkle \& Poppe, 1994). Therefore, the present study investigated comparing $\mathrm{HCl}$ and TFA to consider which acid performed better on hydrolyzing EPS. The results of the study might create a premise for further studies on the chemical structure of EPS.

\section{Materials and methods}

\subsection{Removing protein and obtaining EPS}

Added 20 UI Flavourzyme solution in phosphate buffer $\left(\mathrm{Na}_{2} \mathrm{HPO}_{4} .7 \mathrm{H}_{2} \mathrm{O}\right.$, $\mathrm{NaH}_{2} \mathrm{PO}_{4} . \mathrm{H}_{2} \mathrm{O}, \mathrm{pH}$ 6.5) to concentrated $O$. sinensis culture at a ratio of $1: 5(\mathrm{v} / \mathrm{v})$ at $50^{\circ} \mathrm{C}$ for 2 hours and stirred every 15 minutes. Added a Sevag (chloroform: n-butanol = 2: $1(\mathrm{v} / \mathrm{v})$ ) mixture to the above solution at a ratio of $1: 1(\mathrm{v} / \mathrm{v})$, shook well for 15 minutes, and left for 15 minutes. Then centrifuged at $5000 \mathrm{rpm}$ water for 10 minutes and collected the supernatant. Repeated 5 times. Added $96^{\circ}$ ethanol to the supernatant in a 3:1 ratio (v/v), refrigerated at $4{ }^{\circ} \mathrm{C}$ for 24 hours, collected precipitate and dried (Vo, 2018).

\subsection{Hydrolyzing EPS with acid hydrochloric $(\mathrm{HCl})$}

The reaction was carried out as described by Yu et al. (2002). Added 250mg of EPS to $6 \mathrm{~mL}$ of $10 \% \mathrm{HCl}$ (the number of moles of acid is 40 times the number of moles of EPS converted to glucose) in a closed container of inert argon gas at $70^{\circ} \mathrm{C}-80^{\circ} \mathrm{C}$ for three hours. 
After the reaction, allowed to cool to room temperature, neutralized with $1 \mathrm{M} \mathrm{NaOH}$, and spin to remove water, then checked the product by thin-layer chromatography and high-performance liquid chromatography.

\subsection{Hydrolyzing EPS with acid trifluoroacetic (TFA)}

The reaction was carried out as described by Yang et al. (2009). Added 35mg of EPS to $0.8 \mathrm{~mL}$ of TFA (50 times the number of moles of acid EPS converted to glucose) diluted with $1.7 \mathrm{~mL}$ of distilled water in a closed container of inert argon gas at a temperature of $70^{\circ} \mathrm{C}-80^{\circ} \mathrm{C}$ for 3 hours. After the reaction, cooled to room temperature, neutralized to $\mathrm{pH} 7$ with $1 \mathrm{M} \mathrm{NaOH}$, evaporated to remove water, and then checked hydrolysis products with thin-layer chromatography and high-performance liquid chromatography.

\subsection{Acetylation of hydrolyzed EPS}

Hydrolysis product was added to anhydrous sodium acetate (moles 5 times the number of moles EPS converted to glucose) and acetic hydride (moles 12 times the number of moles EPS converted to glucose) in the closed system at $80^{\circ} \mathrm{C}-90^{\circ} \mathrm{C}$ for 2 hours, then added $20 \mathrm{ml}$ of ethyl acetate (repeated 3 times), collected and concentrated the ethyl acetate phase. Finally, the derivatives were checked by thin-layer chromatography and gas chromatography.

\subsection{HPLC}

HPLC was performed as described in BP 2014 standard. The sample was filtered through a filter paper with a pore size of $0.22 \mu \mathrm{M}$. The conditions of performing HPLC: mobile phase: purified water; column: sugar CMP, particle size $9 \mu \mathrm{M}, 7.8$ x $300 \mathrm{mM}$; flow rate: $0.5 \mathrm{~mL} / \mathrm{min}$; column temperature: $80^{\circ} \mathrm{C}$; injection volume: $20 \mu \mathrm{L}$. Performed with sucrose, glucose, mannose, xylose at a time.

\section{6. $G C$-FID}

GC-FID was performed as described by Yuan et al. (2016). After acetylation, the product was analyzed by GC-FID with the condition: The temperature of the injection chamber, probe temperature: $230^{\circ} \mathrm{C}$. The starting temperature of the column: $110^{\circ} \mathrm{C}$ and stayed for 1 minute before rising to $180^{\circ} \mathrm{C}$. Finally, it would be increased to $280^{\circ} \mathrm{C}$ at $20^{\circ} \mathrm{C} / \mathrm{min}$ and kept for 10 minutes.

\section{Results and discussion}

\subsection{Results of the thin-layer chromatography and HPLC of EPS after hydrolysis}

Under the effect of acid, polysaccharide hydrolyzed into smaller carbohydrate molecules by breaking down these Glycosidic bonds. The results from the chromatogram showed that the pre-hydrolyzed EPS sample has no streaks (Figure 1, track 1). 


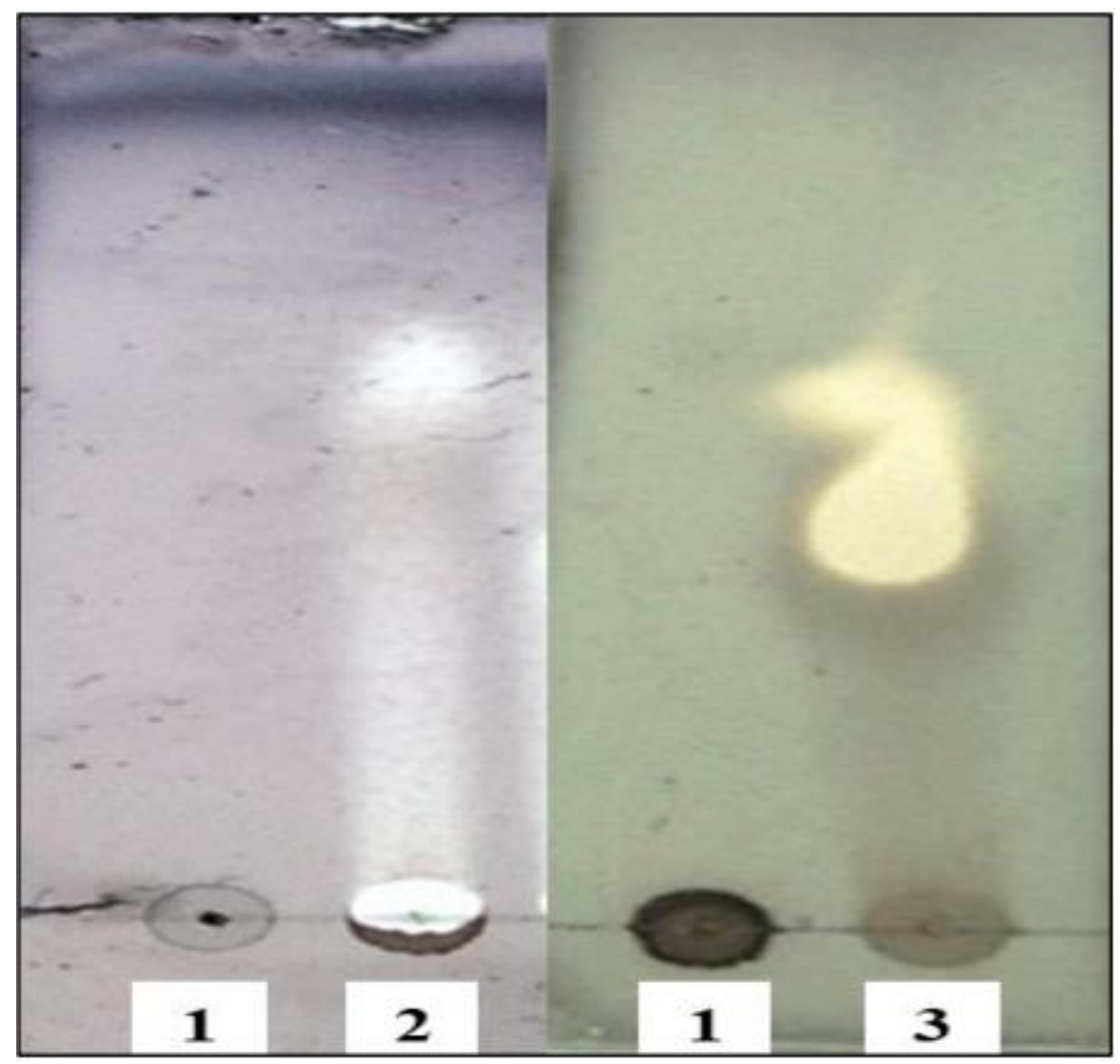

Figure 1. Results of the Thin-layer chromatogram of samples: 1. EPS before hydrolysis, 2 . EPS hydrolyzed with $\mathrm{HCl}, 3$. EPS hydrolyzed with TFA

For hydrolyzed samples with $\mathrm{HCl}$ (Figure 1, track 2), the thin-layer chromatography result showed that a long dark stain was found on the track; therefore, the EPS sample was hydrolyzed. However, the hydrolysis process with this $\mathrm{HCl}$ occurred incompletely, so that the stain was prolonged. The EPS sample after hydrolysis with TFA (Figure 1, track 3) showed a dark stain and a dark spot above the track, which indicated that the EPS sample was hydrolyzed by TFA. The solvent system of Chloroform: methanol: water at the ratio of 10:9:1 has a high degree of polarization so that the streaks on track 2 and track 3 could be the presence of mono, di-, and oligosaccharide which were the product from hydrolysis of EPS samples.

The results of the thin - layer chromatography and HPLC of post-hydrolyzed samples also showed that $\mathrm{HCl}$ exhibited better hydrolysis ability than TFA during EPS hydrolysis. 


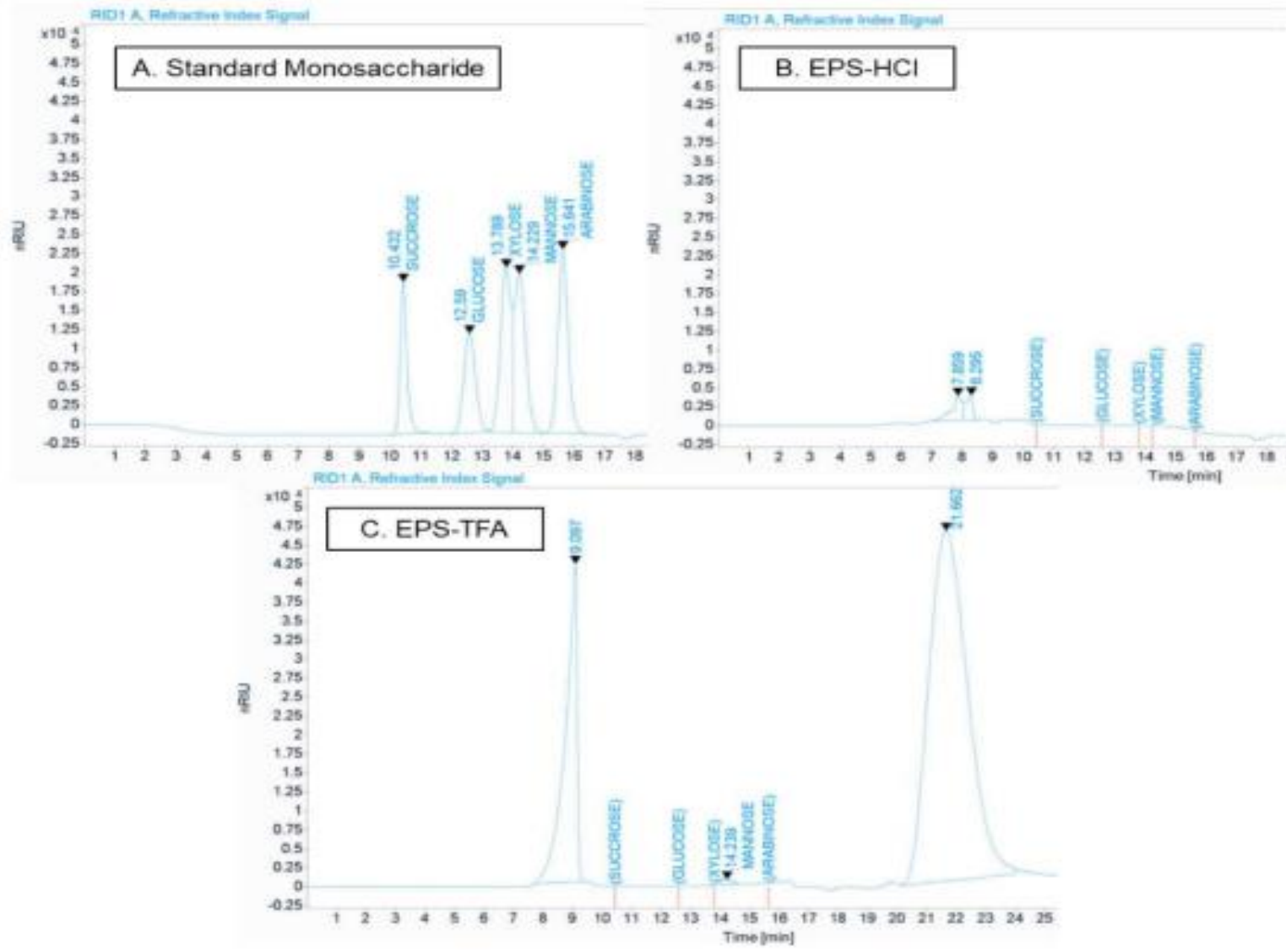

Figure 2. HPLC graphs of samples: A. Standard substance, B. EPS hydrolyzed with HCl, C. EPS hydrolyzed with TFA

HPLC graphs of EPS hydrolyzed with $\mathrm{HCl}$ showed that there were two peaks corresponding to the retention time of 7.859 minutes $(59.14 \%)$ and 8.95 minutes $(40.86 \%)$, none of these peaks matched 5 standard substances (saccharose, glucose, xylose, mannose, and arabinose) (Figure 2B). However, the retention time of these two peaks was very close to Sucrose's retention time. Two signal peaks contributed to prove that $\mathrm{HCl}$ had hydrolyzed EPS.

For EPS samples after hydrolysis by TFA, HPLC graph presented 3 peaks with the retention time of 9.097 minutes (for $21.56 \%$ ), 14.239 minutes (for mannose - $0.40 \%$ ) and 21.662 minutes (for $78.04 \%$ ) which had the retention time longer than that of standard monosaccharide (Figure 2C). The signal peak located near the retention time of mannose peak indicated that the hydrolysis reaction could separate polysaccharide to monosaccharide levels. Most substances were at the peak of 9,097 minutes and 21.662 minutes, which have retention times smaller and longer than the retention time of the five standard sugars. This could happen that a portion of carbohydrate still existed at multi-sugar forms which had not been fully cleaved, whereas a large amount of monosaccharide separated from EPS might have worked with acid to form other substances.

\subsection{Results of thin-layer chromatography of EPS samples after acetylated hydrolysis}

The results of the thin-layer chromatography showed that the EPS hydrolyzed by $\mathrm{HCl}$ was acetylated. The thin-layer chromatogram showed a trace on track 2 (Figure 3), compared 
to the hydrolyzed EPS sample but not acetylated (track 1, no signal of the streak). The solvent system of hexane: chloroform at the ratio of 7:3 has low polarity so that there were the streaks on track 2 possibly indicating the presence of acetylated monosaccharides which were various low-polarity derivatives.

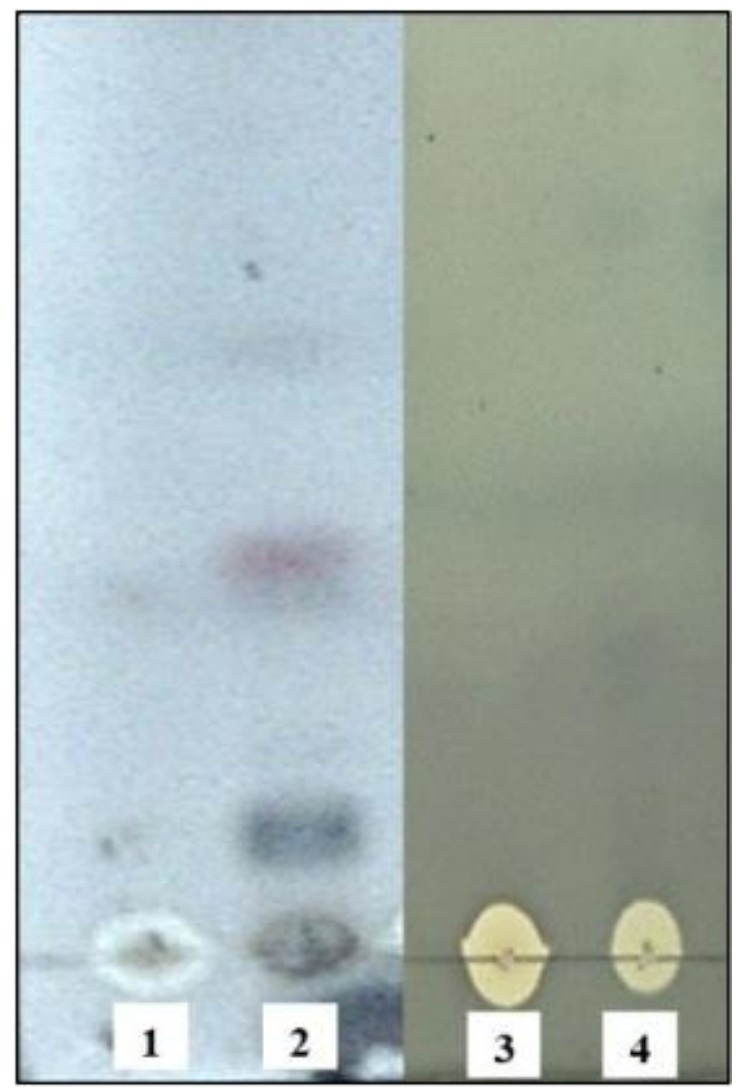

Figure 3. Thin-layer chromatography of samples: 1. HCl-hydrolyzed EPS non-acetylated, 2. HCl-hydrolyzed EPS acetylated, 3. TFA-hydrolyzed EPS non-acetylated, 4. TFA-hydrolyzed EPS acetylated

For EPS hydrolyzed by TFA, the thin-layer chromatographic (Figure 3, track 4) showed very light streaks, the streak at the baseline was still very dark, including the appearance of the white spot. This result suggested that TFA might not completely hydrolyze the EPS sample. The white spots on the baseline presented that there were at least two different substances in the EPS sample after acetylation, in particular, the white spot substance was non-acetylated which had high polarity; therefore, it stayed at the baseline at the end of acetylation.

The results of thin-layer chromatography of samples after acetylation presented that the EPS treated by $\mathrm{HCl}$ was well hydrolyzed and facilitated the great acetylation reaction.

\subsection{Results of GC-FID}

For EPS hydrolyzed by $\mathrm{HCl}$, the analytical results presented that this sample had 6 kinds of monosaccharides, including rhamnose, arabinose, xylose, mannose, glucose, and galactose; the most monosaccharide was glucose with 3.5-fold mannose and 3.1-fold galactose. The EPS hydrolyzed by TFA only detected three kinds of monosaccharides, including mannose, arabinose, and galactose, mainly mannose (Figure 4). 


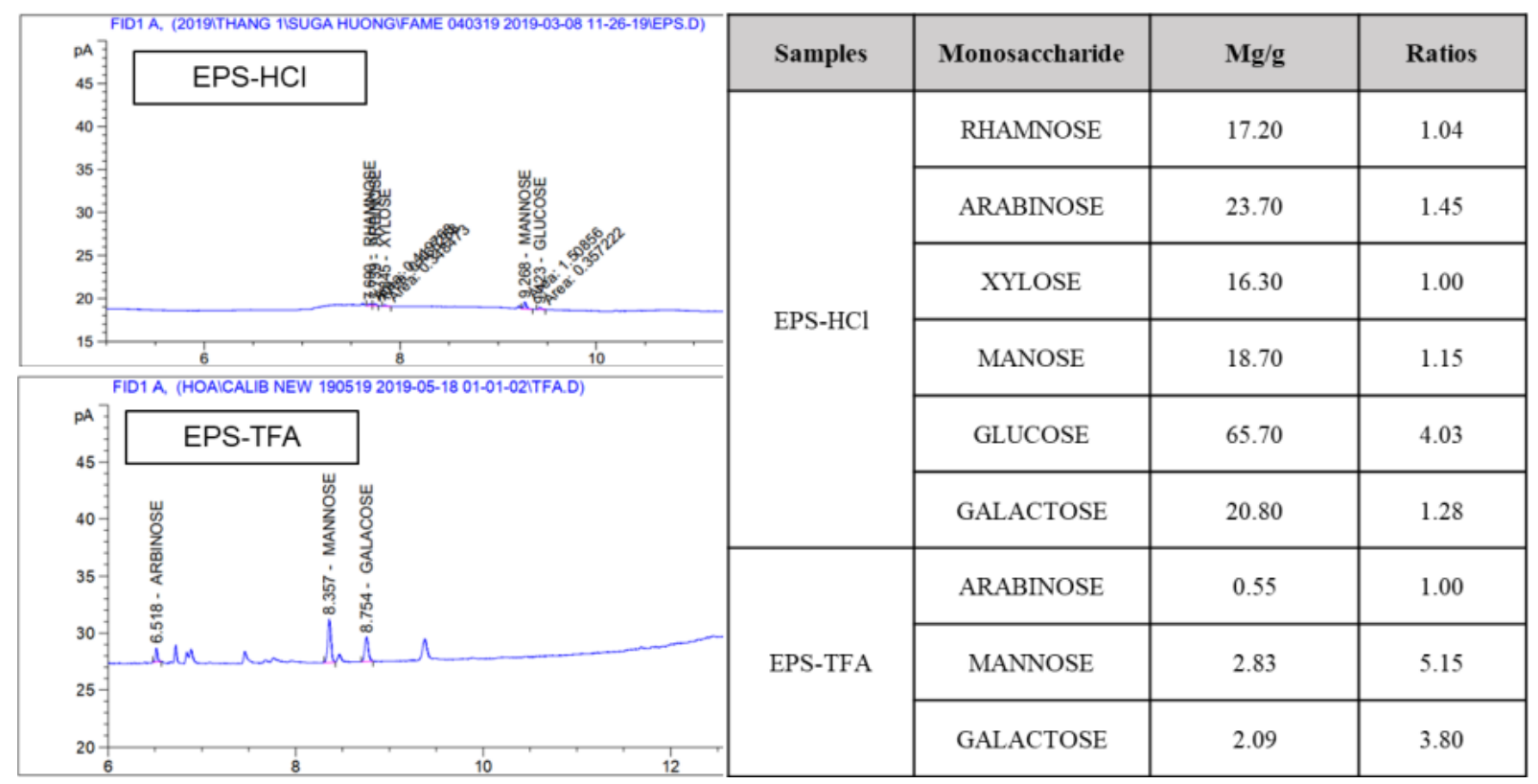

Figure 4. GC-FID graph and monosaccharide composition results of samples:

A. EPS hydrolyzed with $\mathrm{HCl}, \mathrm{C}$. EPS hydrolyzed with TFA

Many kinds of EPSs have been detected and collected, the majorities of their monosaccharide composition are generally glucose, mannose, and galactose in various proportions, in which glucose has been the most monosaccharide (Zhang, Yang, Chen, Hou, \& Han, 2005). In the results of the present study, the result of the monosaccharide composition of EPS hydrolyzed by $\mathrm{HCl}$ was also consistent with the results of Cha et al. (2007) when analyzing the simple sugars of exo-biopolymer from $O$. sinensis 16 mainly including glucose, mannose, and galactose at the ratio of 61.5: 18.1: 9.4; in which glucose was 3-fold mannose and galactose. A study by Vo (2018) on analyzing the monosaccharide composition of EPS segments from $O$. sinensis by HPLC also had the parallel results which showed that the monosaccharides in the EPS segments were mainly mannose, galactose, and glucose, in which glucose was the most monosaccharide.

\section{Conclusions}

EPS hydrolyzed by $\mathrm{HCl}$ had 6 kinds of monosaccharides, including rhamnose, arabinose, xylose, mannose, glucose, and galactose; the most monosaccharide was glucose with 3.5-fold mannose and 3.1-fold galactose. The EPS hydrolyzed by TFA only detected three kinds of monosaccharides, including mannose, arabinose, and galactose, mainly mannose.

The results from the current study indicated that $\mathrm{HCl}$ was suitable for $O$. sinensis exopolysaccharide hydrolysis, pre-treatment before analyzing the monosaccharide composition by GC-FID method, compare with TFA.

\section{References}

Cha, S. H., Lim, J. S., Yoon, C. S., Koh, J. H., Chang, H. I., \& Kim, S. W. (2007). Production of mycelia and exo-biopolymer from molasses by Cordyceps sinensis 16 in submerged culture. Bioresource Technology, 98(1), 165-168. 
Hon, D. N. S. (2001). Cellulose: Chemistry and technology. In K. H. J. Buschow, R. W. Cahn, M. C. Flemings, B. Ilschner, E. J. Kramer, S. Mahajan \& P. Veyssière (Eds.), Encyclopedia of materials: Science and technology (pp. 1039-1045). Oxford, UK: Elsevier.

Lo, H. C., Hsieh, C., Lin, F. Y., \& Hsu, T. H. (2013). A systematic review of the mysterious caterpillar fungus ophiocordyceps sinensis in Dong-ChongXiaCao (Dong Chong Xia Cao) and related bioactive ingredients. Journal of Traditional and Complementary Medicine, 3(1), 16-32.

Merkle, R. K., \& Poppe, I. (1994). Carbohydrate composition analysis of glycoconjugates by gas-liquid chromatography/mass spectrometry. Methods in Enzymology, 230, 1-15.

Nie, S. P., Cui, S. W., \& Xie, M. Y. (2018). Cordyceps polysaccharides. In S. Nie, S. W. Cui \& M. Xie (Eds.), Bioactive polysaccharides (pp. 143-204). London, UK: Academic Press.

Selvendran, R. R., March, J. F., \& Ring, S. G. (1979). Determination of aldoses and uronic acid content of vegetable fiber. Analytical Biochemistry, 96(2), 282-292.

Soltani, M., Kamyab, H., \& El-Enshasy, H. A. (2013). Molecular weight (Mw) and monosaccharide composition (MC): Two major factors affecting the therapeutic action of polysaccharides extracted from cordyceps sinensis. Journal of Pure and Applied Microbiology, 7(3), 1601-1613.

Vo, T. P. T. (2018). Study on methods of obtaining exopolysaccharide fractions from Ophiocordyceps sinensis culture (Master's thesis). VNUHCM-University of Science, Ho Chi Minh city, Vietnam.

Yan, J. K., Wang, W. Q., \& Wu, J. Y. (2014). Recent advances in Cordyceps sinensis polysaccharide: Mycelial fermentation, isolation, structure, and bioactivities: A review. Journal of Functional Foods, 6, 33-47.

Yang, B., Yu, G., Zhao, X., Jiao, G., Ren, S., \& Chai, W. (2009). Mechanism of mild acid hydrolysis of galactan polysaccharides with highly ordered disaccharide repeats leading to a complete series of exclusively odd-numbered oligosaccharides. The FEBS Journal, 276(7), 2125-2137.

Yu, G., Guan, H., Ioanoviciu, A. S., Sikkander, S. A., Thanawiroon, C., \& Tobacman, J. K. (2002). Structural studies on k-carrageenan derived oligosaccharides. Carbohydrate Research, 337, 433-440.

Yuan, Y., Wang, Y. B., Jiang, Y., Prasad, K. N., Yang, J., Qu, H., .. Yang, B. (2016). Structure identification of a polysaccharide purified from Lycium barbarium fruit. International Journal of Biological Macromolecules, 82, 696-701.

Zhang, W., Yang, J., Chen, J., Hou, Y., \& Han, X. (2005). Immunomodulatory and antitumour effects of an exopolysaccharide fraction from cultivated Cordyceps sinensis (Chinese caterpillar fungus) on tumour-bearing mice. Biotechnology and Applied Biochemistry, 42(1), 9-15. 